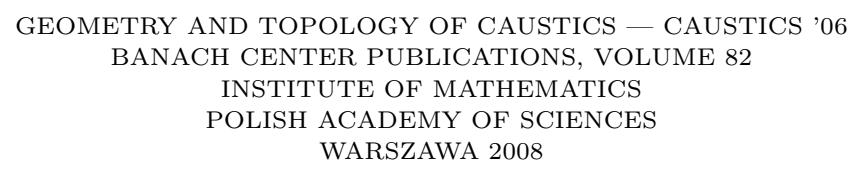

\title{
SURFACES WHICH CONTAIN MANY CIRCLES
}

\author{
NOBUKO TAKEUCHI \\ Department of Mathematics, Tokyo Gakugei University \\ Koganei-shi, Tokyo, 184-8501, Japan \\ E-mail:nobuko@u-gakugei.ac.jp
}

\begin{abstract}
We survey the results on surfaces which contain many circles. First, we give two analyses of shapes which always look round. Then we introduce the Blum conjecture: "A closed $C^{\infty}$ surface in $E^{3}$ which contains seven circles through each point is a sphere", and give some partial affirmative results toward the conjecture. Moreover, we study some surfaces which contain many circles through each point, for example, cyclides.
\end{abstract}

1. Introduction. This article is a survey on the results about surfaces which contain many circles. "A sphere" is a familiar shape. Most people would describe a sphere as a round object. Conversely, is a shape which appears round from any angle always a sphere? In order to answer this question, we first give two analyses of shapes which always look round. Then we see a sphere contains many circles on it. But we do not know a surface other than a sphere or a plane, which contains an infinite number of circles through each point of it. We would like to know the minimum number of circles through each point of the surface which determines its shape to be a sphere or a plane. Moreover we give some surfaces which contain many circles through each point.

\section{Two analyses of shapes which always look round}

Part I. If someone standing in any position is able to see the object in question as a circular form, then one may decide the object is a sphere.

2000 Mathematics Subject Classification: 53A05, 53A30.

Key words and phrases: sphere, circle.

The paper is in final form and no version of it will be published elsewhere. 


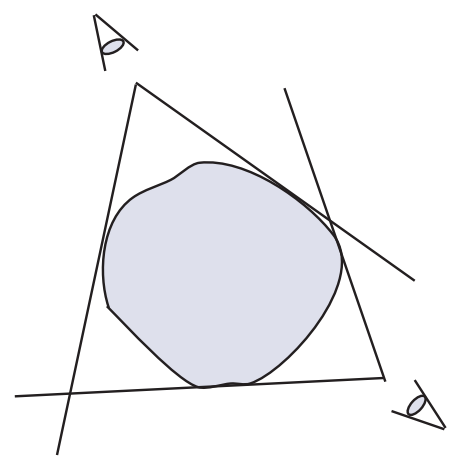

Circular cones may be used to distinguish whether the object in question is in fact a circular form. Then the vertex of the cone may be applied at every angle. One may also use circular cylinders, if the object in question is examined from a distance.

We know that an object which only looks round from limited positions may not be called a sphere, such as an ellipsoid of revolution.

Professor Shigetake Matsuura dealt with this problem in his 1980-1981 articles (in Japanese) published in the Tokyo-based Japanese mathematical journal called Suugaku seminar. He concluded that only shapes which appear circular from every angle are spheres.

Part II. If a circle in $E^{3}$ of any given radius can be pasted perfectly in any position on the object in question, then one may decide the object is a sphere.
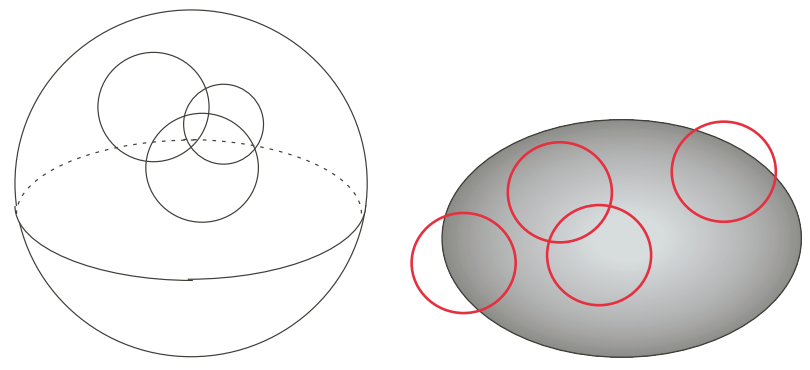

During the war, the Japanese military used cannon balls. The balls needed to be perfect spheres, that is, objects which may be viewed as circular from any angle in order to function properly. If they were not perfect spheres, they would fail to hit their target. Only perfect spheres proved to be useful weapons. Subsequently, the military developed a methodology for examining the shapes of the newly made balls. They would paste multiple circles onto the surface of the ball in question. If a circle could be placed on any part of the ball's surface and fit properly, they would conclude that the ball was a perfect sphere and thus include it in their weaponry supplies. 


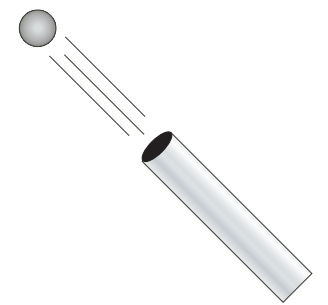

We know that a sphere in $E^{3}$ is characterized as a closed surface which contains an infinite number of circles in $E^{3}$ through each point. But we do not know a surface other than a sphere or a plane, which contains many circles through each point of it.

In the following figures, $(n)$ is the number of circles through a point $P$ on the surface.

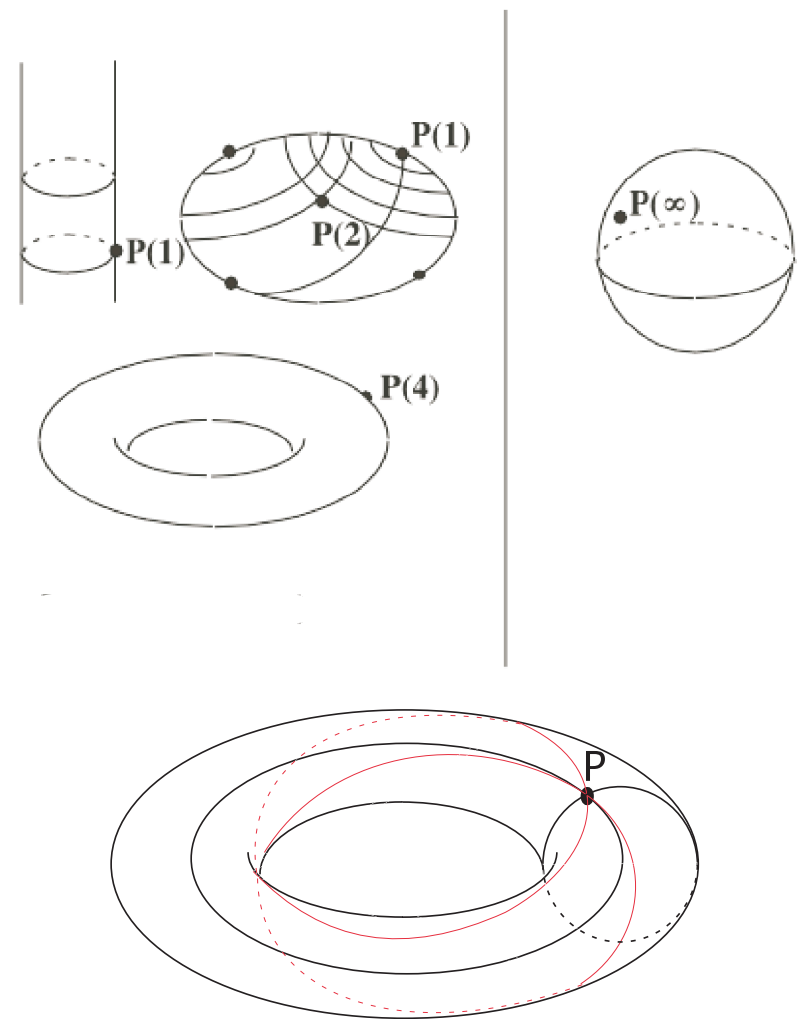

3. Conjectures and theorems. In 1980, Richard Blum [1] found a closed $C^{\infty}$ surface of genus one which contains six circles through each point, and he gave a conjecture:

Conjecture 1 (R. Blum [1]). A closed $C^{\infty}$ surface in $E^{3}$ which contains seven circles through each point is a sphere.

However he did not produce original affirmative theorems for this conjecture.

In 1984, Koichi Ogiue and Ryoichi Takagi [2] showed the following theorem: 
Theorem 1 (K. Ogiue and R. Takagi [2]). A $C^{\infty}$ surface in $E^{3}$ is (a part of) a plane or a sphere if it contains two circles through each point, which are tangent to each other.

Additionally, drawing from the fact that an ellipsoid contains two circles through each point except only at four points, they gave the following conjecture:

Conjecture 2 (K. Ogiue and R. Takagi [2]). A simply connected complete $C^{\infty}$ surface in $E^{3}$ is a plane or a sphere if it contains two circles through each point.

We have the following partial affirmative results toward Conjectures 1 and 2:

TheOREM $2([5])$. A simply connected complete $C^{\infty}$ surface in $E^{3}$ is a plane or a sphere if it contains three circles through each point.

TheOREm $3([5]) . A C^{\infty}$ surface in $E^{3}$ is (a part of) a plane or a sphere if it contains three circles through each point, any two of which are tangent to each other or have two points in common.
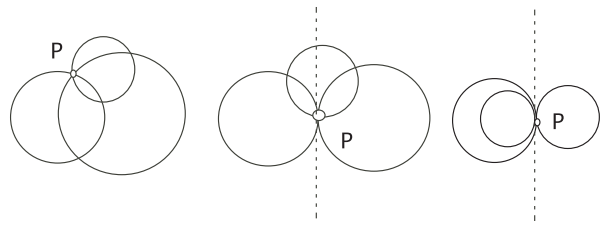

TheOrem $4([6])$. A closed $C^{\infty}$ surface of genus one in $E^{3}$ cannot contain seven circles through each point.

Our next theorem usefully demonstrates the theories of our forefathers in their testing of the functionality of cannon balls used during the war.

Theorem 5 (K. Ogiue and N. Takeuchi [4]). A smooth ovaloid in $E^{3}$ is a sphere if the surface contains a circle of an arbitrary but fixed radius through each point.

\section{Examples of surfaces which contain many circles}

EXAMPLE 1 (Hulahoop surfaces [3]). A hulahoop surface is a smooth surface obtained by revolving a circle around a suitable axis.
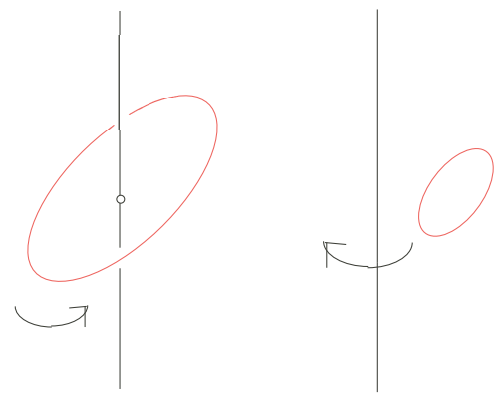

Let $\gamma(a, b, r), r>0$ be a circle on the $x y$-plane defined by

$$
(x-a)^{2}+(y-b)^{2}=r^{2}
$$


and let $\gamma(a, b, r, \alpha)$ be the circle obtained by tilting $\gamma(a, b, r)$ around the diameter parallel to the $x$-axis by the angle $\alpha,-\frac{\pi}{2}<\alpha \leq \frac{\pi}{2}$. It is easily seen that $\gamma(a, b, r, \alpha)$ is given by

$$
\begin{aligned}
& x=a+r \cos \theta, \\
& y=b+r \cos \alpha \sin \theta, \\
& z=r \sin \alpha \sin \theta .
\end{aligned}
$$

Let $H(a, b, r, \alpha)$ be the surface obtained by rotating $\gamma(a, b, r, \alpha)$ around the $z$-axis. Then it is easily seen that $H(a, b, r, \alpha)$ is a smooth surface if and only if $a=b=0$ and $\alpha=\frac{\pi}{2}$ or $a \neq 0$ and $\left(a^{2}-r^{2}\right) \cos ^{2} \alpha+b^{2} \neq 0$. We see that $H\left(0,0, r, \frac{\pi}{2}\right)$ is a sphere and otherwise $H(a, b, r, \alpha)$ is topologically a torus. Note that $H(a, b, r, \alpha)$ contains at least two circles through each point, one is a latitudinal circle and the other is a rotated $\gamma(a, b, r, \alpha)$. We denote $\gamma(a, b, r, \alpha) \sim \gamma(\bar{a}, \bar{b}, \bar{r}, \bar{\alpha})$ when two circles are congruent under the rotation around the $z$-axis. For example, $\gamma(a, b, r, \alpha) \sim \gamma(-a,-b, r,-\alpha)$. It is clear that if $\gamma(a, b, r, \alpha) \sim \gamma(\bar{a}, \bar{b}, \bar{r}, \bar{\alpha})$, then $H(a, b, r, \alpha)=H(\bar{a}, \bar{b}, \bar{r}, \bar{\alpha})$. We see that $H(a, b, r, \alpha)$ is obtained by revolving the curve on the $x z$-plane defined by

$$
\begin{aligned}
& x=\sqrt{(a+r \cos \theta)^{2}+(b+r \cos \alpha \sin \theta)^{2}}, \\
& y=0, \\
& z=r \sin \alpha \sin \theta,
\end{aligned}
$$

and hence that $H(a, b, r, \alpha)$ is defined by the equation

$$
\begin{array}{r}
\left(x^{2}+y^{2}+z^{2}\right)^{2}-\frac{4 b \cos \alpha}{\sin \alpha}\left(x^{2}+y^{2}+z^{2}\right) z-2\left(a^{2}+b^{2}+r^{2}\right)\left(x^{2}+y^{2}\right) \\
-2\left(a^{2}+b^{2}+r^{2}-\frac{2 a^{2}+2 b^{2} \cos ^{2} \alpha}{\sin ^{2} \alpha}\right) z^{2}+\frac{4 b \cos \alpha}{\sin \alpha}\left(a^{2}+b^{2}+r^{2}\right) z \\
+\left(a^{2}+b^{2}+r^{2}\right)^{2}-4 a^{2} r^{2}=0
\end{array}
$$

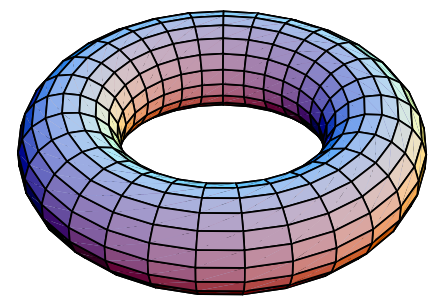

Then, we can see a hulahoop surface which is not a sphere contains exactly four or five circles through each point.

THEOREM 6 ([3]). A compact smooth surface of revolution which contains at least two circles through each point is a hulahoop surface.

COROLlaRY 7 ([3]). There exists no compact smooth surface of revolution which contains exactly $k$ circles through each point for $k=2,3,6,7, \ldots$. 
Corollary 8 ([3]). A compact smooth surface of revolution which contains exactly four circles through each point is an ordinary torus.

EXAMPLE 2 (Blum's surface [1]). R. Blum's surface defined by a quartic equation of the form:

$$
\left(x_{1}^{2}+x_{2}^{2}+x_{3}^{2}\right)^{2}-2 a_{1} x_{1}^{2}-2 a_{2} x_{2}^{2}-2 a_{3} x_{3}^{2}+a=0 \quad\left(a_{1} \geq a_{2}>0, a_{3}<-\sqrt{a}\right)
$$

They contain exactly four (if $a_{1}=a_{2}, a_{3}=-\sqrt{a}$ ), five (if $a_{1}=a_{2}, a_{3} \neq-\sqrt{a}$, or if $a_{1} \neq a_{2}, a_{3}=-\sqrt{a}$ ) or six (if $a_{1} \neq a_{2}, a_{3} \neq-\sqrt{a}$ ) circles through each point.

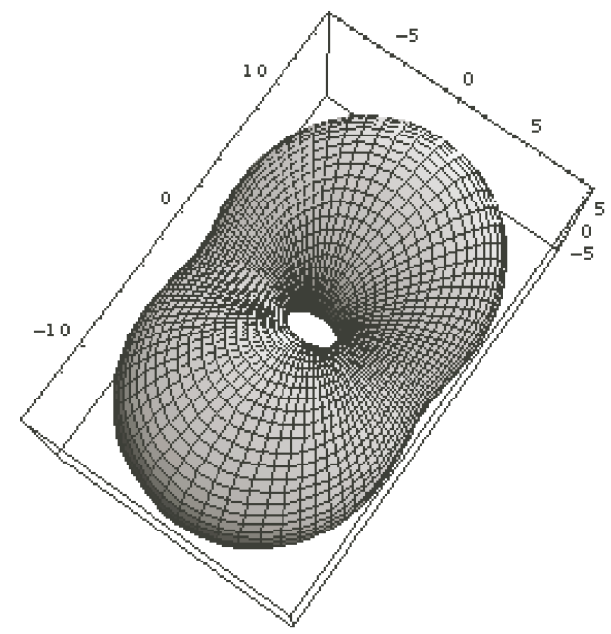

Furthermore, we must recognize the fact that cyclides contain many circles.

ExAmple 3 (Cyclides [7]). A cyclide is a surface in $E^{3}$ defined by a quartic equation of the form:

$$
\left(x_{1}^{2}+x_{2}^{2}+x_{3}^{2}\right)^{2}+2\left(x_{1}^{2}+x_{2}^{2}+x_{3}^{2}\right) \sum_{i=1}^{3} b_{i} x_{i}+\sum_{i, j=1}^{3} a_{i j} x_{i} x_{j}+2 \sum_{i=1}^{3} a_{i} x_{i}+a=0
$$

An ordinary torus gives a typical example and quadratic surfaces are considered as singular examples. A closed $C^{\infty}$ surface of genus one which contains six circles through each point, which R. Blum had found, is also one of cyclides.

Subsequently we developed the following theorems.

THEOREM $9([7])$. A non-singular cyclide is conformally equivalent to a cyclide of the form:

$$
\left(x_{1}^{2}+x_{2}^{2}+x_{3}^{2}\right)^{2}-2 a_{1} x_{1}^{2}-2 a_{2} x_{2}^{2}-2 a_{3} x_{3}^{2}+a=0(a \neq 0)
$$

which is topologically a torus, a sphere or two spheres. A cyclide with singularities is conformally equivalent to a quadratic surface.

THEOREM 10 ([7]). A cyclide contains $n$ circles through each non-umbilic point and $n-1$ circles through each isolated umbilic point unless it is a sphere or a pair of two spheres, where $n=1,2,3,4,5$ or 6 . 

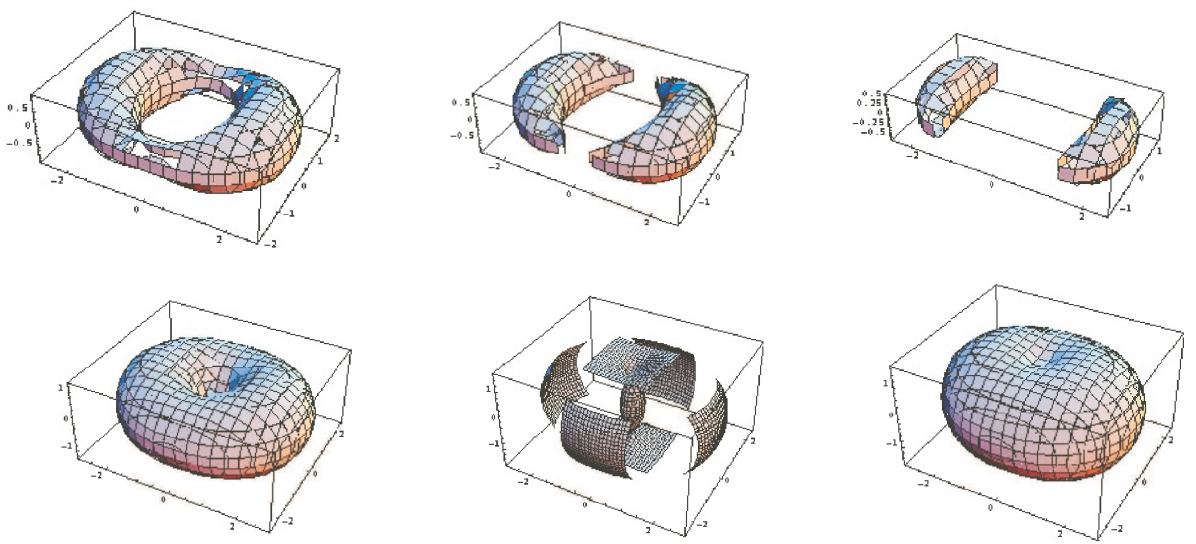

\section{References}

[1] R. Blum, Circles on surfaces in the Euclidean 3-space, in: Geometry and Differential Geometry, Lecture Notes in Math. 792, Springer, Berlin, 1980, 213-221.

[2] K. Ogiue and R. Takagi, A submanifold which contains many extrinsic circles, Tsukuba J. Math. 8 (1984), 171-182.

[3] K. Ogiue and N. Takeuchi, Hulahoop surfaces, J. Geom. 46 (1993), 127-132.

[4] K. Ogiue and N. Takeuchi, A sphere can be characterized as a smooth ovaloid which contains one circle through each point, J. Geom. 49 (1994), 163-165.

[5] N. Takeuchi, A sphere as a surface which contains many circles, J. Geom. 24 (1985), $123-130$.

[6] N. Takeuchi, A closed surface of genus one in $E^{3}$ cannot contain seven circles through each point, Proc. Amer. Math. Soc. 100 (1987), 145-147.

[7] N. Takeuchi, Cyclides, Hokkaido Math. J. 29 (2000), 119-148. 
